\title{
LED based near infrared spectral acquisition for multiwavelength analytical ultracentrifugation: A case study with gold nanoparticles
}

\author{
Joseph Pearson, Helmut Cölfen* \\ Physical Chemistry, University of Konstanz, Universitätsstraße 10, 78457 Konstanz, Germany
}

H I G H L I G H T S

- NIR spectral analysis now possible with analytical ultracentrifugation.

- Pulsed LEDs provide NIR illumination for multiwavelength AUC.

- Gold nanoparticle distributions and spectral properties may be recovered.

- Method applicable to a broad range of plasmonic and semi-conductor nanoparticles.
G R A P H I C A L A B S T R A C T

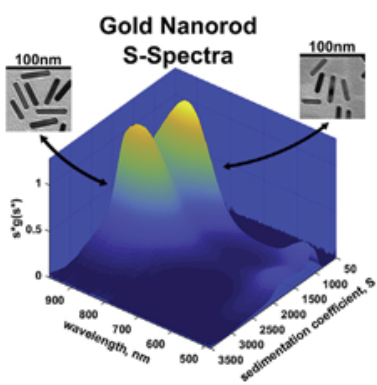

A B S T R A C T

A pulsed light emitting diode, coupled with a properly configured spectrometer is implemented in open source analytical ultracentrifugation detection hardware, allowing for spectral acquisition spanning the visible to near infrared. Details of the challenges arising with the new hardware are described, along with the necessary solutions to overcome them. The system is successfully tested with polystyrene and gold nanoparticles, demonstrating effectiveness for different material and particle geometries. Sedimentation coefficient distributions and analytically extracted spectra are presented for the multiwavelength datasets extending into the near infrared. Additionally, deconvolution algorithms are applied as a further demonstration of tools applicable to these datasets and the resolving power possible. The results of gold nanoparticle analysis presented here show effective near infrared spectral acquisition is now possible for multiwavelength analytical ultracentrifugation, opening the door for analysis of many important samples with spectral properties in this range, including rich classes of metal and semiconductor nanoparticles.
Analytical ultracentrifugation

Near infrared

Multiwavelength

Gold nanorods

Gold nanoparticles

\section{Introduction}

\subsection{Nanoparticle analysis by ultracentrifugation}

Colloidal gold sols have been studied extensively in recent decades. Vast array of scientific investigations have probed the synthesis mechanisms giving rise to a wide variety of shapes and sizes, and possessing interesting chemical and photonic properties. Research into gold nanoparticle dispersions has been the subject of numerous recent reviews [1-6]. Interest in the underlying 
properties of gold nanoparticle solutions has historic origins and received particular attention in the early years of the 20th century. One notable historical work, and especially relevant to the subject of this manuscript, is the research of Svedberg, the originator of analytical ultracentrifugation (AUC). Svedberg selected as his first subject of study colloidal gold sols. In recent years, AUC has shown resurgence in use for the investigation of nanoparticles in solution [7-12]. The usefulness of AUC in these endeavors has been expanded by the development of multiwavelength (MWL) absorbance optical systems. A large body of work has chronicled the advances in MWL-AUC instrumentation, beginning with the first construction in the Cölfen laboratory [13], and published as open source materials as part of the OpenAUC project [14,15]. The potential for MWL-AUC has drawn particular attention to the study of metallic and semiconductor nanoparticles, owing to the extraordinary size and shape dependent optical properties at the nano scale. AUC has the capability to resolve nanoparticle size distributions with Angstrom resolution, and with MWL detection, to also probe spectral properties in dispersion [16,17].

Gold nanorods (AuNRs) show especially striking spectral properties, arising from the localized surface plasmon resonance phenomena operating in the transverse (TSPR) and longitudinal (LSPR) rod dimensions. The LSPR extinction band is remarkably sensitive to the aspect ratio of the AuNRs, and spans from the visible (Vis) through the near infrared (NIR) region of the spectrum. Spectral properties of AuNRs have been the subject of extensive theoretical inquiry, and several methods exist for modeling the spectral properties of AuNRs in solution [18-24]. The applicability of the various methods is complicated by the difficulty in repeatable synthesis techniques to produce AuNRs of uniform size and axial ratio. A large body of work has gone into developing protocols for the synthesis of AuNRs with low size and shape dispersity [24,25]. Centrifugation has been proposed as an effective purification method for gold nanoparticles of different size and shape [6]. While MWL-AUC has been proven as a valuable tool for studying nanoparticle dispersions and spectral property distributions, a MWL optical system capable of covering the Vis through NIR has remained a challenge. In this manuscript we present a novel adaptation to MWL-AUC, allowing for the detection of spectra signals spanning the Vis to NIR, and apply this method to a case study of gold nanoparticle dispersions.

\subsection{Multiwavelength analytical ultracentrifugation instrumentation}

Modern analytical ultracentrifugation absorbance detectors capture spectral signals from the UV through the Vis. All of the existing and emerging technology of the last several decades is based on illumination with a Xenon flash lamp. A Xenon flash lamp emits high UV-Vis luminance over very short pulse duration. The short pulse and fast repetition rate allows synchronization with an AUC rotor spinning at $60,000 \mathrm{rpm}$. A photomultiplier tube, such as is implemented in the commercial Beckman Coulter XL-A, is in principle fast enough to provide synchronization, however, they are not implemented in this way, because even a high power constant source lamp would likely not provide enough light for the short duration necessary to capture a sample channel passing the detector at high velocity. The radiant power of a Xenon pulse is much higher than a constant illumination source, even with significantly higher rated Watts, over an equivalent duration. With the spectrometer-based detector design, as implemented in the Open AUC MWL architecture, a limitation also arises from the charge transfer rate of the CCD chip. The minimum integration time of an Ocean Optics spectrometer is $1 \mathrm{~ms}$, and even the fastest CCD camera available is in the tens of micro seconds. A sample channel spinning at $60,000 \mathrm{rpm}$ will pass the detector aperture in approximately $4 \mu$ s However, there are also several disadvantages imposed by a Xenon flash lamp. The flash-to-flash repetition exhibits intensity fluctuations that are the limiting contribution to maximizing the signal-to-noise ratio (SNR). Also, the luminance of a Xenon lamp is extremely non-linear with high emission spikes in the UV and Vis, while decreasing significantly into the NIR. The Ocean Optics spectrometer typically used in the MWL instrument contains an "XR" grating with a practical limit in the NIR of $650 \mathrm{~nm}$. This can be extended slightly further by so-called high sensitivity acquisition modes, but comes at the expense of making the Vis spectral region unusable [15]. It is important to note; the recorded intensity spectrum is always a function of the wavelength variant power output from the source, and the wavelength dependent sensitivity of the detector. For the Ocean Optics USB2000 + spectrometer, the sensitivity of the linear CCD chips (Sony ILX511) has a maximum at approximately $450 \mathrm{~nm}$.

Previous attempts have been made on the MWL hardware to compensate for the low NIR output of the Xenon flash lamp by implementing a spectrometer configured to optimize for NIR signals [26]. The construction of a NIR USB2000 + spectrometer from Ocean Optics is achieved by selection of a diffraction grating that is generated with a blaze wavelength in the NIR, and positioned to focus a NIR bandwidth onto the spectrometer CCD chip. The NIR spectrometer implementation was shown to provide NIR absorbance signals from the AUC, and a valid sedimentation coefficient distribution was achieved from selected NIR wavelengths, for a sample of gold nanorods with NIR extinction bands. However, the limited spectral signals from the samples were shown. Follow up measurements with this spectrometer system revealed strange spikes in the absorbance data in the NIR. These spikes were also in the raw data from Ref. [26], but were not mentioned, and wavelength signals from regions without spikes were used for analysis. When an absorbance spectrum of a sample with NIR extinction bands is plotted along with a reference intensity spectrum taken with the NIR spectrometer and Xenon flash lamp, the origin of the data spikes become readily apparent; see Fig. 1.

The high intensity emission lines at discrete NIR wavelengths line up exactly with the apparent spikes in the absorbance spectrum. The likely explanation for this is because the recorded signals are saved as integer wavelength spectra, where several pixels from the spectrometer chip may be averaged. If a single pixel is depleted, but an adjacent pixel still has significant charge, then the average value of the two will still be below the apparent saturation limit of the detector. An additional complication of this configuration is the absence of an order-sorting filter in the NIR spectrometer as supplied from Ocean Optics. Order sorting filters in this type of spectrometer serve to block higher order diffraction wavelengths from superimposing on the same pixels of the spectrometer chip. To observe the effect, a $500 \mathrm{~nm}$ high pass filter was introduced between the source and detector; see Fig. 1. The resulting loss of intensity in the NIR is in accordance with what might be anticipated from 2nd order wavelengths, where the efficiency of the grating is typically $20 \%$ less than for the 1 st order [27].

The NIR spectrometer configuration (Ocean Optics NIR spectrometer with \#14 grating, $600 \mathrm{l} / \mathrm{mm}$, blazed at $1.0 \mu \mathrm{m}$, with a bandwidth of 383-1106 nm) is not typically possible to buy with an order-sorting filter at this time. However, Ocean Optics was able to build one as a special-order device. The only caveat is that the useable bandwidth was limited to $1000 \mathrm{~nm}$. Test results using the special order NIR spectrometer and a Xenon flash lamp show that the relative signal available in the NIR is very limited with this configuration Fig. 2. Attempting to compensate for the low NIR signal by using a high sensitivity acquisition mode caused the Vis region of the spectrum to saturate the detector, in addition to 
several of the high emission spikes in the NIR. An example reference intensity, and corresponding absorbance spectrum possible with this system is shown in Fig. 2, along side an absorbance spectrum of the same sample taken with a constant illumination Deuterium-Tungsten lamp (Ocean Optics USBDT). It is clear this configuration is unusable for broad spectrum Vis-NIR MWL AUC experiments. To overcome these limitations a new system design is constructed based on an array of pulsed light emitting diodes (LEDs). The LED array is selected to generate a balanced Vis-NIR spectrum with pulse width timing adjusted for rotor synchronization. LEDs can be pulsed fast enough to serve as a triggered light source in AUC, and were first reported for use with AUC Schlieren optics more than two decades ago [28].

\section{Materials and methods}

\subsection{LED based NIR illumination}

High power LEDs in a heat sink housing integrated with fiber optic SMA couplers are available from Thorlabs with a large selection of wavelength outputs, including a broadband Vis option. Coupling a selection of several LEDs allows for generation of a broadband illumination source producing light through the VisNIR. Emission intensity spectral data is available from Thorlabs for the different LED models, allowing simulation of a combined spectral profile Fig. 3.

Multimode fan-out fiber bundles, also available from Thorlabs, accomplish coupling of the LEDs. The individual legs of the fiber optic cables attach to the SMA adapter coupling of the LED housings, and the opposing fiber ends are configured within a single SMA adapter, where the fiber cores are assembled into a tight bundle. For this system, an item\# BF42LS01; 4 Fan-out, MM, SMA, $200 \mu \mathrm{m}, 0.39 \mathrm{NA}, 1 \mathrm{~m}$, fiber bundle is used.

Four LEDs were selected with nominal wavelength outputs of $780 \mathrm{~nm}, 850 \mathrm{~nm}, 940 \mathrm{~nm}$, and a broadband LED with 450-870 nm output.

Several LED driver options are available from Thorlabs, including

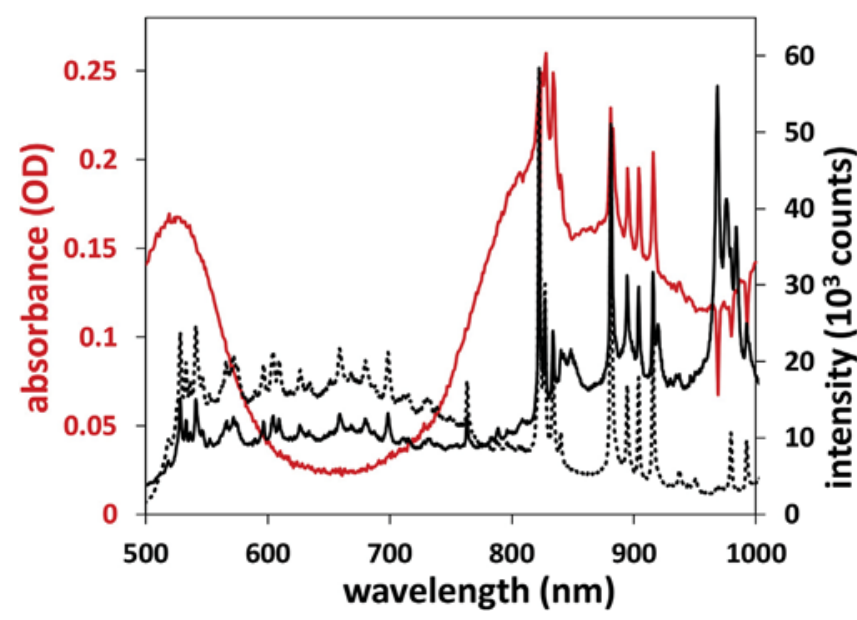

Fig. 1. Demonstration of second-order gratings effects present in an Ocean Optics NIR spectrometer without order sorting filter: (Black line) Reference intensity spectrum of an Ocean Optics NIR spectrometer with \#14 grating, $600 \mathrm{l} / \mathrm{mm}$, blazed at $1.0 \mu \mathrm{m}$, with a bandwidth of 383-1106 nm, and a Hamamatsu L9455-13 Xenon flash lamp. (Red line) Absorbance spectrum of a gold nanorod sample acquired with the same reference intensity shows the effects of higher-order grating signals. (Dotted black line) Reference intensity spectrum of identical configuration, but with a $500 \mathrm{~nm}$ high pass filter inserted between the illumination source and detector. (For interpretation of the references to color in this figure legend, the reader is referred to the Web version of this article.)

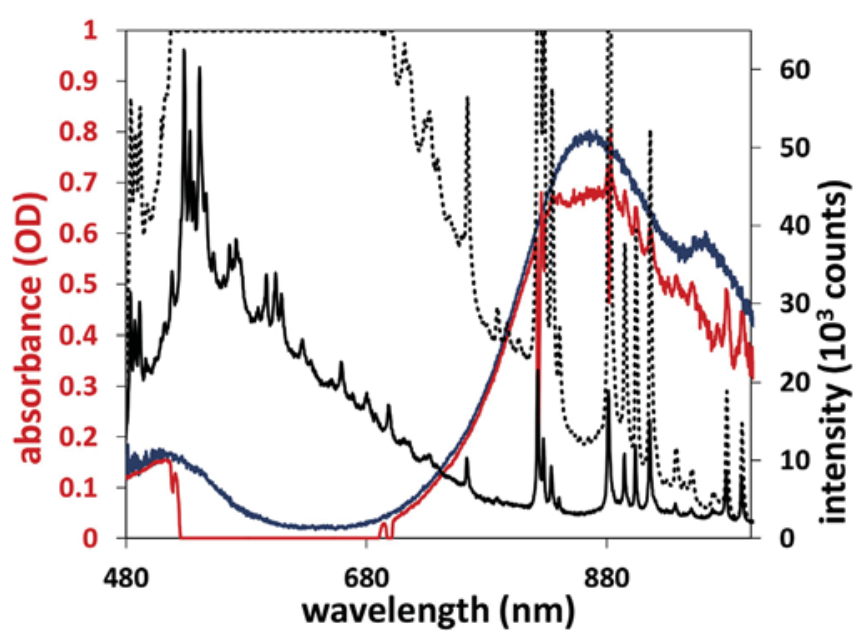

Fig. 2. Demonstration of limitations with a Xenon flash lamp and Ocean Optics spectrometer with order sorting filter: (Black line) Reference intensity spectrum from a special-order Ocean Optics NIR spectrometer (\#14 grating, $600 \mathrm{l} / \mathrm{mm}$, blazed at $1.0 \mu \mathrm{m}$, bandwidth 383-1106 nm, and including an order-sorting filter), and a Hamamatsu L9455-13 Xenon flash lamp. (Dotted black line) Reference intensity spectrum from an identical configuration is shown using a high sensitivity acquisition mode accumulating three flashes per acquisition. (Red line) Absorbance spectrum of a gold nanorod sample acquired with a high sensitivity acquisition mode reference intensity, showing the limitations of Xenon emission with NIR absorbance signals. (Blue line) Absorbance spectrum of identical gold nanorod sample acquired with a constant illumination Deuterium-Tungsten lamp (Ocean Optics USBDT), reference spectrum not shown. (For interpretation of the references to color in this figure legend, the reader is referred to the Web version of this article.

single channel and 4 channel models. The single channel model is known to be slower than the 4-channel model. The LEDD1B driver specifies a rise time of $51 \mu \mathrm{s}$ and fall time of $79 \mu$ s The DC2200 driver is much faster with rise and fall times less than $5 \mu \mathrm{s}$, dependent on current levels and load (Thorlabs correspondence). However, the DC2200 is more expensive and limited to 4 channels. A custom-built driver was constructed by the Universität Konstanz Electronic Service Center. Circuit information is included in the Appendix; Supporting Information. The IRF540 chips provide very fast response times. Exact LED rise and fall times would require specialized test equipment, and be dependent on current levels, similar to the DC2200. Furthermore, using drivers constructed as individual circuits, allows the possibility to add additional channels as needed. The dimensions of the chip layout on the printed circuit boards allowed for two drivers with equivalent current limitation to be connected in parallel to the same power supply. The drivers are triggered by connection with coaxial BNC cables to the typical National Instruments hardware used in the MWL system. An image of the LEDs, drivers and fiber bundle assembly is provided in the Appendix; Supporting Information.

Modulating the intensity profile from the individual LEDs could be achieved by including additional circuitry, but may also be accomplished by adjustment of the LED to fiber coupling. The common end of the 4-fiber bundle was connected to an additional $500 \mu \mathrm{m}$ core fiber segment by SMA couplers. The output end of the fiber segment was then installed in the MWL optical detector arm, with a slit adapter on the end of the fiber entering the illumination optics. The lens-based focused illumination and lens-based imaging architecture was used, as it is known to have higher transmission [15]. Furthermore, chromatic aberration concerns of lens based systems are known to be more extreme for the UV spectral region and mostly problematic for fitting data sets to sedimentation and diffusion models [15]. Therefore the limitations of the lens-based system described previously will also apply to the NIR signals 


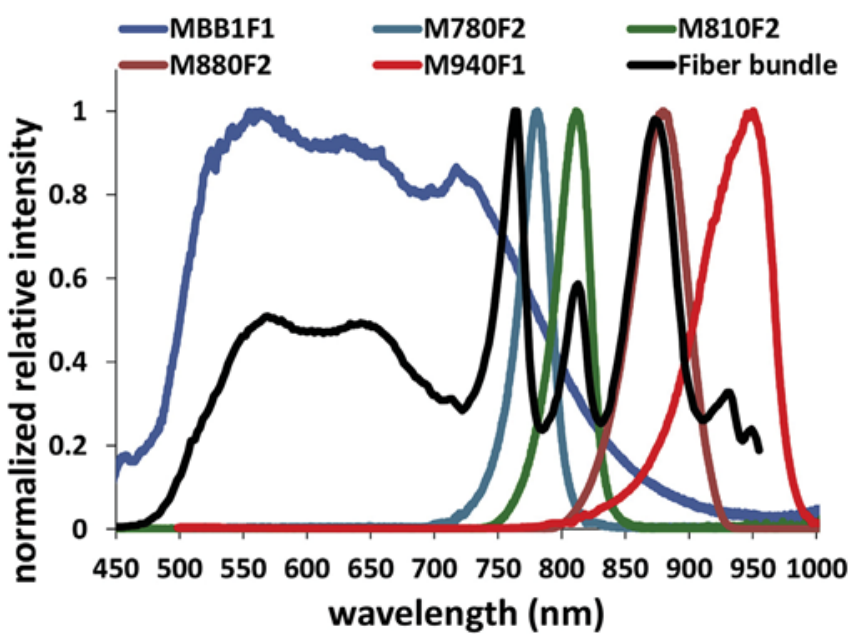

Fig. 3. Relative spectral emission profile from individual Thorlabs NIR fiber coupled LEDs, provided with permission from the Thorlabs catalog. (Black line) Emission profile of LEDs combined into a single fiber bundle and measured through the MWL AUC optics with an Ocean Optics NIR spectrometer; \#14 grating, 600 l/mm, blazed at $1.0 \mu \mathrm{m}$, with a bandwidth of 383-1106 $\mathrm{nm}$ and order sorting filter. A peak emission wavelength offset is observed with the M780F2 LED compared with the manufacturer specifications. The manufacturer notes variations and a current dependency from the catalog emission profiles but does not give a tolerance.

although to a lesser extent. To record sedimentation in the Vis-NIR, without correcting for diffusion, the lens-based detector is fully adequate. A first test spectrum of the 4-fiber bundle contained a region between 790 and $820 \mathrm{~nm}$ of low intensity. This was partly due to the $780 \mathrm{~nm}$ LED being blue shifted from the wavelength specified in the catalog. One additional $810 \mathrm{~nm}$ LED was included by attaching a secondary fiber splitter to one leg of the 4-fiber bundle, and combining the $780 \mathrm{~nm}$ and $810 \mathrm{~nm}$ LEDs into the single leg. The relative emission of the 780 and $810 \mathrm{~nm}$ LEDs was higher than the other LEDs, so despite the additional coupling, still allowed for enough intensity. A spectral intensity profile of the coupled LED array installed in the MWL detector is shown in Fig. 3, with the model numbers of the respective LEDs.

The primary challenge of successfully implementing this system for NIR-MWL-AUC experiments is that LED power output is much weaker than the Xenon flash lamp traditionally employed. A dual strategy is needed to overcome the limited signal availability. First; the high sensitivity software acquisition mode, accumulating multiple LED pulses in a single acquisition is essential [15]. The second requirement, pulse width modulation, is unique to this implementation. Because LEDs will emit light in response to a positive driver signal, extending the time the signal to the driver remains positive may modulate the duration of a pulse. This is in contrast to the Xenon flash lamps that emits with fixed pulse durations of approximately $2 \mu \mathrm{s}$, regardless of trigger pulse width.

The width of the LED driver signal must be set to match the time it takes for the sample channel to pass below the detector optics. The sector shaped sample channel has a width of $2.5^{\circ}$, while the spectrometer entrance slit is $1 \mathrm{~mm}$ long in the corresponding dimension, leaving a difference of approximately $1.52^{\circ}$ across the channel. A simple rotor speed dependent calculation may then be used to estimate the 'open-window' time that the $1 \mathrm{~mm}$ spectrometer entrance slit may capture optical signals through the channel. For a rotor spinning at $3000 \mathrm{rpm}$ this equates to approximately $84 \mu \mathrm{s}$. The open window time will be inversely correlated with increasing rotor speeds. For effective execution of this configuration, a careful angle calibration is required such that the illumination pulse rise and fall is adequately synchronized with the open window time.

\subsection{Experimental data validation}

The example data presented in the following section focuses on nanoparticles with relatively large sedimentation coefficients. Therefore, all data is collected at a low rotor speed of $3000 \mathrm{rpm}$. At the time of the experiments, a vacuum chamber feed through for the fiber optic cable was not immediately available; therefore the test data demonstrating the proof of principle of the detector system were carried out under atmospheric conditions. The centrifuge is able to operate without vacuum in 'zonal' mode up to $3000 \mathrm{rpm}$. The data is then converted to $S_{20 w}$ conditions (equivalent to water at $20^{\circ} \mathrm{C}$ ), for comparison.

All AUC measurements were made using the high sensitivity acquisition mode with 5 accumulations. While the signal intensity of the combined LED array is more balanced than what is typically seen in MWL AUC with a Xenon lamp, there is still variability at the emission maxima of the LEDs, and in between. The average signal measured across the Vis-NIR spectrum is 14778 counts, and provides a SNR of 207 at this signal intensity. No significant variability in SNR is seen in the measured spectral region but declines precipitously below $500 \mathrm{~nm}$ and above $950 \mathrm{~nm}$. Therefore the practical bandwidth for acquisitions with this system is $480-960 \mathrm{~nm}$. An abnormal jump in intensity is observed occurring within the first scan of a run, likely due to the LEDs warming up. Therefore, the first scan in the data sets is dropped before analysis. It is important to note that the LEDs and drivers were observed to be strongly affected by external electromagnetic fields. Proper shielding from the laboratory AC power is essential. Test scans at $3000 \mathrm{rpm}$, where the rotations matched the $50 \mathrm{~Hz}$ period of the AC laboratory power were particularly vulnerable to inducing strange signal oscillations when made without grounded metal shielding.

To prove the basic validity of the recorded signals, a first test sample of polystyrene nanospheres was analyzed (Duke Scientific, Cat No. 3100A, Mean NIST traceable diameter $102 \mathrm{~nm} \pm 3 \mathrm{~nm}$ ). The sample was diluted to $\sim 1$ OD at $525 \mathrm{~nm}$, and run in titanium double sector centerpiece cells at $3000 \mathrm{rpm}$. The AUC control was set to the temperature of the laboratory and the rotor was allowed to stabilize for $1 \mathrm{~h}$ prior to starting; $24^{\circ} \mathrm{C}$, measured with an infrared thermometer. The AUC recorded temperature fluctuations were less than $0.5^{\circ} \mathrm{C}$ over the course of the experiment.

The results were evaluated with SedAnal version 668, using the Wide Distribution Analysis (WDA) [29]. The WDA method transforms the experimental data into a sedimentation coefficient distribution, uncorrected for diffusion, termed $\mathrm{g}\left(\mathrm{s}^{*}\right)$. For fast sedimenting particles, i.e. $>100 \mathrm{~S}$, this method is appropriate, as very little diffusion signal is available in the data to attempt fitting with models including diffusion. The apparent weighted sedimentation coefficient $\left(\mathrm{s}^{* \mathrm{w}}\right)$ is taken from an integral selection of the $\mathrm{g}\left(\mathrm{s}^{*}\right)$ distribution, encompassing the full width half maximum of the signal peak, and may be corrected to the value at standard conditions $\left(\mathrm{s}_{20, \mathrm{w}}\right)$ in the usual way [30]. Values are reported in units of Svedbergs (S) along with the integral bounds used in weighted average estimations. The $\mathrm{s}^{* \mathrm{~W}}$ is used to estimate the hydrodynamic particle diameter, $d_{p}$, by the well-known particle sizing equation [31], with the assumption of spherical particle geometry.

Equation (1);

$d_{p}=\sqrt{\frac{18 \cdot \eta \cdot s^{*} w}{\left(\rho_{p}-\rho_{s}\right)}}$

where $\eta$ is the solvent viscosity $(0.9107 \mathrm{cp}), \rho_{\mathrm{s}}$ is the solvent density $\left(0.9973 \mathrm{~g} / \mathrm{cm}^{3}\right)$, and $\rho_{\mathrm{s}}$ the density of the particle $\left(1.05 \mathrm{~g} / \mathrm{cm}^{3}\right)$. The $\mathrm{s}^{* \mathrm{~W}}$ measured with the MWL-NIR enabled AUC detector was $349 \mathrm{~S}$ (280-420), and results in $\mathrm{d}_{\mathrm{p}}=104 \mathrm{~nm}$; within the tolerance 
provided by the manufacturer of the Nanosphere sample.

Experiments with slower sedimenting particles that would require higher rotor speeds have yet to be explored but are in principle feasible by a prudent selection of a shorter pulse width and increased number of accumulated flashes. For fast sedimenting and polydisperse samples a speed ramp method would be useful, as a larger number of samples can be analyzed in a single experiment, and a higher dynamic range of particle sizes probed. However, in such a case the pulse width, flash accumulation setting and spectrometer integration time would need to be dynamically modulated throughout the experiment. This is not realized in the current software design, but the possibility of this option is being explored. The present implementation, in sedimentation velocity mode, is restricted to two cells so as to limit scan times to $130 \mathrm{~s}$.

\section{Results}

\subsection{Gold nanorods case study}

Having confirmed the accuracy of the measurement system with well characterized polystyrene nanospheres; we next demonstrate the usefulness of this detector for the evaluation of plasmonic gold nanoparticles, showing interesting spectral properties extending into the NIR. Two gold nanorod (AuNR) samples, synthesized by a seeded growth method, were provided by Susanne Seibt, Universität Bayreuth. The two samples, designated $S_{A}$ and $S_{B}$ were measured with a Zeiss Libra 120 transmission electron microscope (TEM) at the University of Konstanz, providing length and diameter distributions $\mathrm{L}_{\mathrm{A}}=48.2 \pm 7.8 \mathrm{~nm}, \mathrm{~d}_{\mathrm{A}}=9.1 \pm 0.8 \mathrm{~nm}$, $\mathrm{L}_{\mathrm{B}}=35.9 \pm 4.8 \mathrm{~nm}, \mathrm{~d}_{\mathrm{B}}=8.1 \pm 0.7 \mathrm{~nm}$ approximated from 50 particles manually using iTEM software. TEM images are provided in the Appendix; Supporting Information. Both samples were measured with the MWL-NIR-AUC at $22{ }^{\circ} \mathrm{C}$, and otherwise identical conditions as with the polystyrene nanospheres. Runs were made of the samples individually and then as a mixture. The evaluation by the SedAnal WDA method was carried out for the entire useable spectrum from 480 to $960 \mathrm{~nm}$. The most recent versions of SedAnal feature the possibility to conduct $\mathrm{g}\left(\mathrm{s}^{*}\right)$ analysis on entire MWL datasets and allow the user to exploit the information contained in the spectral dimension of these experiments. Such as spectral deconvolution of the raw sedimentation profiles or spectral extraction of the resolved sedimentation coefficient distributions [12].

In Fig. $4-A$ and $B, g\left(s^{*}\right)$ distributions are shown for $S_{A}$ and $S_{B}$ from two wavelength signals; one from the peak of the LSPR band, and one from the $510 \mathrm{~nm}$ signal typical of the maximum for the TSPR band for AuNRs of this size. A $g\left(s^{*}\right)$ distribution should theoretically result in a Gaussian peak profile for a monodisperse sample. Therefore, the presence of asymmetric tails, or multimodal distributions in a peak profile is indicative of populations of particles significantly different than the primary solute distribution mode. Extracting spectral information from these regions of an $\mathrm{S}$ distribution can yield information about the nature of the secondary distribution modes. For sample $\mathrm{S}_{\mathrm{A}}$, the appearance of a broad tail on the upper and lower side of the $S$ distribution indicates the presence of both additional larger and smaller solutes. While for sample $S_{B}$, there is a tail on only the upper side of the $S$ distribution. The different shape of the tails on the sides of the $S$ distributions for the two wavelength signals, suggest different spectral properties of the subpopulation. To further explore the spectral properties of the subpopulations, spectra data is extracted from the tail regions of the $S$ distributions. This is done by selecting a region of the $S$ distribution tail for an entire MWL data set, from 480 to $960 \mathrm{~nm}$, all analyzed simultaneously by the WDA method. Extracted spectra are shown in Fig. 4 - C and D, with the weighted average sedimentation coefficients and integration bounds for the respective spectral extraction regions. To further illustrate the small spectral shifts observable in the subpopulations, the distributions are normalized to the TSPR peak at approximately $510 \mathrm{~nm}$ in Fig. 4 $E$ and $F$. The differences in the relative extinction ratio of the $510 \mathrm{~nm}$ TSPR peak and the LSPR peak are clearly evident.

Samples $S_{A}$ and $S_{B}$, were then mixed in equal ratio and run under
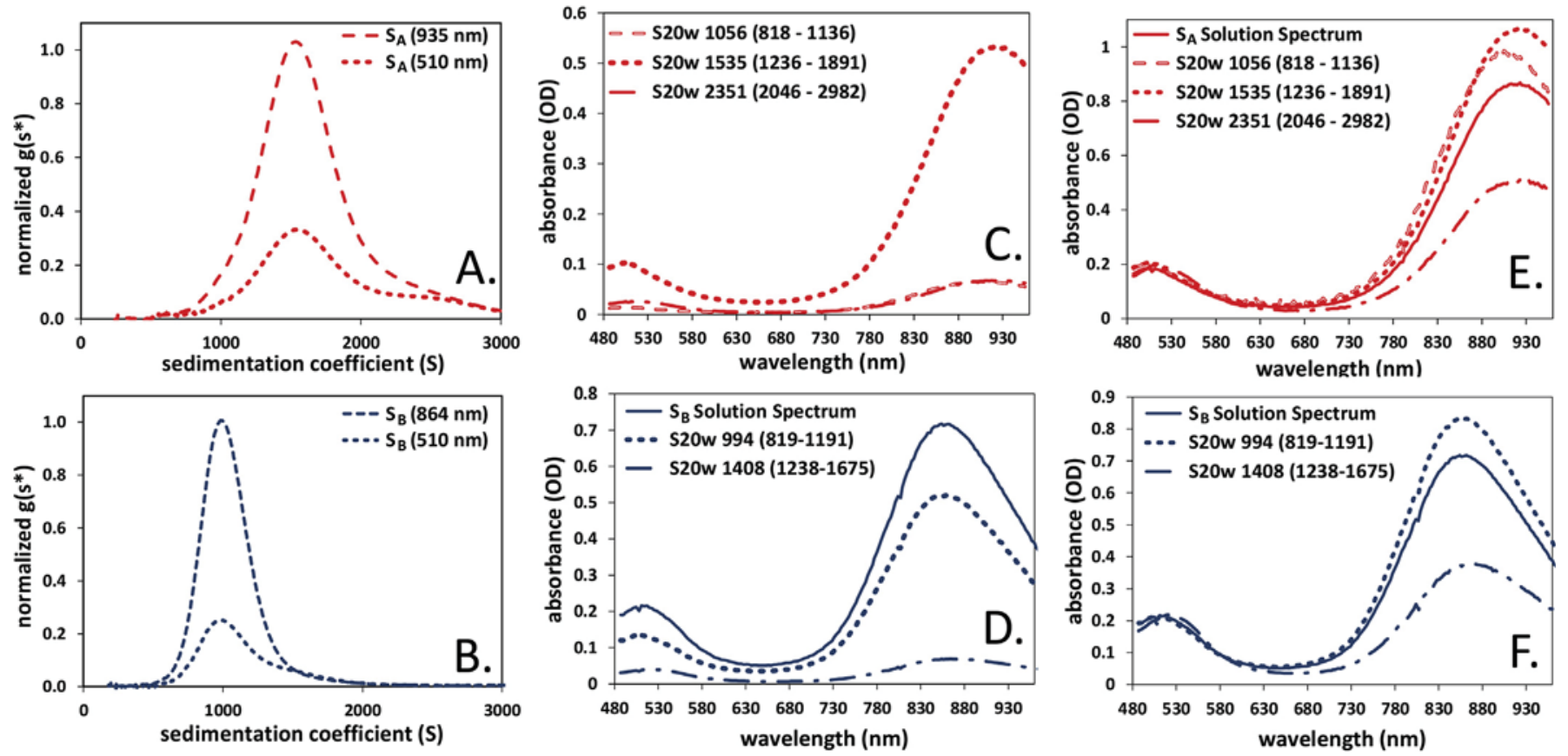

Fig. 4. Results of AuNR samples measured by NIR-MWL-AUC; showing $g\left(s^{*}\right)$ sedimentation coefficient distributions for separate experiments of two AuNR samples, $S_{A}$, in plot $A$., and $S_{B}$ in plot B. Absorbance spectra extracted from the regions of the sedimentation coefficient distributions for $S_{A}$, in plot $C$., and $S_{B}$ in plot $D$. Plots $E$. and $F$. show the same data as plots C. and D., but normalized to the TSPR peak at approximately $510 \mathrm{~nm}$. 

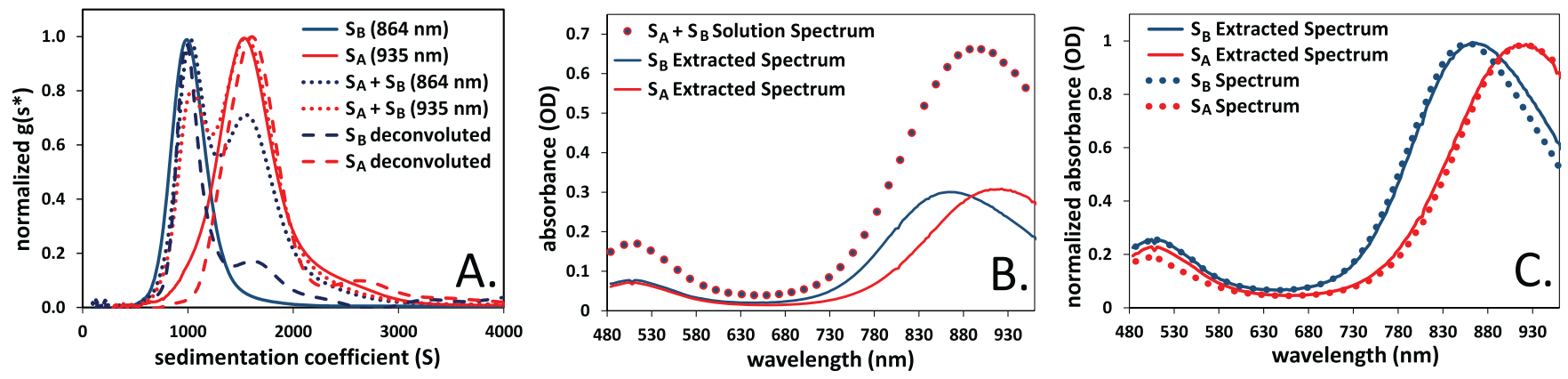

Fig. 5. Results of a mixture of AuNR samples measured by NIR-MWL-AUC; plot A. shows the $g\left(\mathrm{~s}^{*}\right)$ sedimentation coefficient distributions for wavelengths of the LSPR peak of each sample, (dotted red line) $S_{A}$ and (dotted blue line) $S_{B}$. In addition, the $g\left(s^{*}\right.$ ) distributions from the spectrally deconvoluted data sets (dashed red line) $S_{A}$ and (dashed blue line) $S_{B}$ are shown. Included in the same plot are the $g\left(\mathrm{~s}^{*}\right)$ distributions for samples (red line) $S_{A}$ and (blue line) $S_{B}$ run individually. Plot $B$. shows spectra extracted from the two peaks of the bimodal distribution, plotted along with the spectrum of the mixture. Plot C. shows the spectra from plot B., plotted alongside the spectra of the pure samples prior to mixing. (For interpretation of the references to color in this figure legend, the reader is referred to the Web version of this article.)
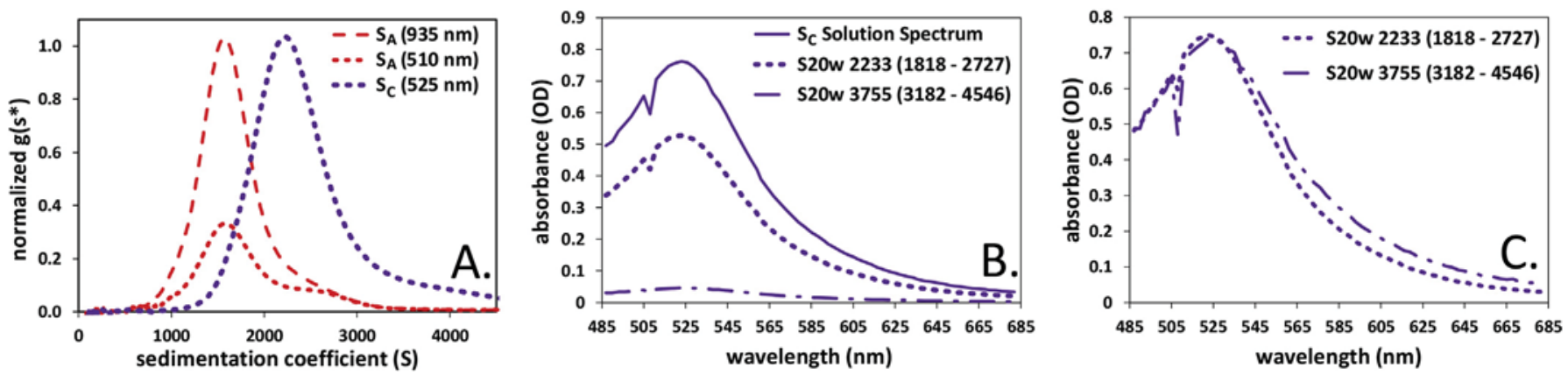

Fig. 6. Results of an AuNS sample, $\mathrm{S}_{C}$, measured by NIR-MWL-AUC; plot A. shows the $\mathrm{g}\left(\mathrm{s}^{*}\right)$ sedimentation coefficient distribution for $\mathrm{S}_{C}$, plotted with the $\mathrm{g}\left(\mathrm{s}^{*}\right)$ distribution of $\mathrm{S}_{\mathrm{A}}$ from the prior experiments. Plot $B$. shows absorbance spectra extracted from the peak and tail regions of the sedimentation coefficient distributions for $S_{C}$. Plot $C$. shows the same data as plot $B$., but normalized to the absorbance peak at approximately $510 \mathrm{~nm}$. Note; the small spike in the spectra around $508 \mathrm{~nm}$ is from an apparently dead pixel in the spectrometer CCD array.

the same conditions as the experiments of the individual samples. The result is a clearly bimodal distribution, shown in Fig. 5 - A. The $\mathrm{g}\left(\mathrm{s}^{*}\right)$ distributions plotted for wavelengths of the LSPR peak of each sample, show the respective maxima of the corresponding rods. The bimodal distribution is plotted along with the individual distributions shown in Fig. 4 - A and B, providing good agreement between the pure and fractionated sedimentation coefficients. The spectra extracted from the two peaks of the bimodal distribution are shown in Fig. 5 - B, and in Fig. 5 - C together with the spectra of the individual samples.

\subsection{Gold rods and spheres mixture}

An additional example experiment with the NIR-MWL-AUC detector, demonstrates a mixture of rods and spheres. A sample, $\mathrm{S}_{\mathrm{C}}$, of gold nanospheres (AuNSs) was prepared by Holger Hilbert at the Universität Konstanz. The diameter distribution was measured with TEM at the University of Konstanz; $\mathrm{d}_{\mathrm{c}}=19.8 \pm 2.05 \mathrm{~nm}$, approximated from 50 particles manually using iTEM software. TEM images are provided in the Appendix; Supporting Information. The AuNS solution was diluted to $0.8 \mathrm{OD}$ at $510 \mathrm{~nm}$, and run, first alone, under the same conditions as the experiments with the AuNRs. The results are shown in Fig. 6 - A, indicating a broad distribution of particles sedimenting significantly faster than $\mathrm{S}_{\mathrm{A}}$. Spectra are extracted from the peak and the tail of the $g\left(\mathrm{~s}^{*}\right)$, and plotted in Fig. 6 - B; normalized in Fig. 6 - C.

Sample $S_{C}$ was then mixed with AuNR sample $S_{A}$, in proportions such that the wavelength maximum at $510 \mathrm{~nm}$ and $900 \mathrm{~nm}$ were both approximately $0.8 \mathrm{OD}$. The mixture was then run in the NIR-
MWL-AUC under the same conditions as the previous experiments. The $\mathrm{g}\left(\mathrm{s}^{*}\right)$ results are shown in Fig. 7 - A, and the extracted spectra in Fig. 7 - B. and C.

As a final analysis, a spectral deconvolution algorithm available in SedAnal is applied to the MWL data set of the $S_{A}$ and $S_{C}$ mixture. The absorbance spectrum of the individual samples are imported as reference spectra, the signal contributions in the mixture from $S_{A}$ and $\mathrm{S}_{\mathrm{C}}$ are then analytically deconvoluted to extract sedimentation scans of the individual solutes. The deconvoluted data sets are then processed with the WDA algorithm in the normal way, and $\mathrm{g}\left(\mathrm{s}^{*}\right)$ distributions produced. The results are included in Fig. 7 - A. The discrepancy between the pure sphere sample and the sphere signal extracted from the mixture is likely due to the sub-population of spheres present in the AuNR sample, $\mathrm{S}_{\mathrm{A}}$, shifting the $\mathrm{s}^{* \mathrm{~W}}$ to a smaller value.

\subsection{Three dimensional $g\left(s^{*}\right)-$ spectral distributions}

The $\mathrm{g}\left(\mathrm{s}^{*}\right)$ distributions, analyzed in parallel in SedAnal, may be subsequently extracted and plotted in 3-dimensions. For this Matlab version R2015a, from The MathWorks, Inc., Natick, Massachusetts, United States is used to illustrate the 3-dimensional 'Sspectra' of the $\mathrm{g}\left(\mathrm{s}^{*}\right)$ distributions with pseudo 3-dimensional plots Fig. 8.

\section{Discussion}

The implementation of fiber bundled LEDs for NIR-MWL-AUC detection technology is introduced, highlighting the principle 

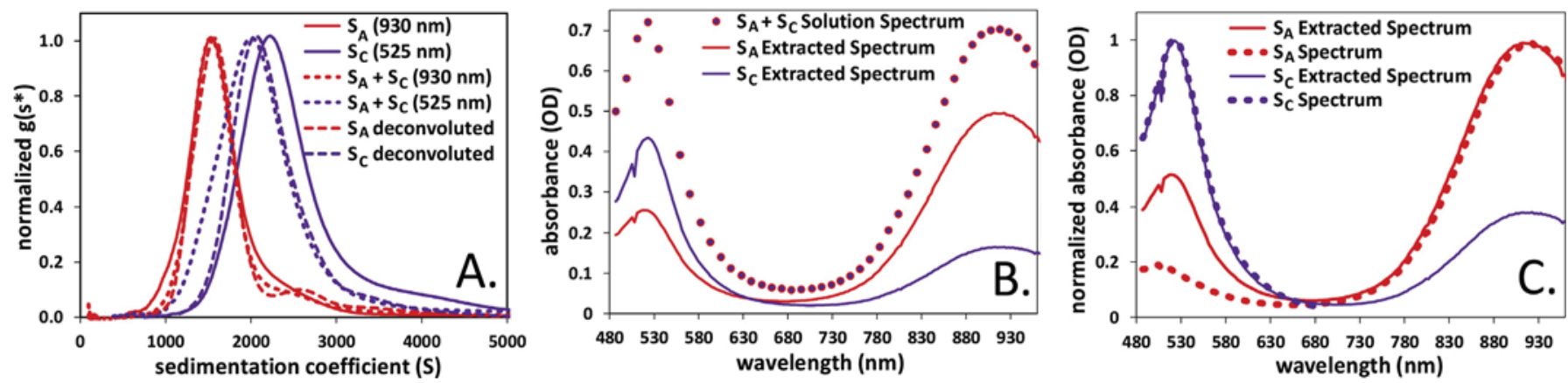

Fig. 7. Results of an AuNR-AuNS mixture measured by NIR-MWL-AUC; plot A. shows the $\mathrm{g}\left(\mathrm{s}^{*}\right)$ sedimentation coefficient distributions for the AuNR-AuNS mixture, plotted for wavelengths of the spectral peak maxima of each sample, (dotted red line) $S_{A}$ and (dotted purple line) $S_{c}$. In addition, the $g\left(s^{*}\right)$ distributions from the spectrally deconvoluted data sets (dashed red line) $S_{A}$ and (dashed purple line) $S_{C}$ are shown, also including $g\left(s^{*}\right.$ ) distributions of (solid red line) $S_{A}$ and (solid purple line) $S_{C}$ alone, plotted previously in Fig. 6 - A. Plot B. shows spectra extracted from the two peaks of the $\mathrm{g}\left(\mathrm{s}^{*}\right)$ distribution, plotted along with the spectra of the mixture. Plot $\mathrm{C}$. shows spectra from plot B., plotted alongside the spectra of the pure samples prior to mixing. (For interpretation of the references to color in this figure legend, the reader is referred to the Web version of this article.)

hardware requirements, such as a properly configured NIR spectrometer. The hardware is able to adapt to the existing MWL optical hardware, and functions with the same operating software, requiring only minimal modification [12,32]. The necessary acquisition mode adaptations are explained, including modulating the LED pulse width to match the open window timing of the sample channel. The basic validity of the data is demonstrated by analysis of a polystyrene nanosphere sizing standard.

An analysis of AuNRs is provided to show the effectiveness of this optical system for particles with extinction spectra extending into the NIR. The tremendous interest in these plasmonic particles for a broad range of applications, amplifies the promise of this new hardware for many areas of research [1-4]. The power of NIRMWL-AUC to fractionate solute populations according to sedimentation behavior, and extract respective spectra is revealed by both a mixture of AuNRs, and AuNRs with AuNSs. Spectra extracted from regions of the $\mathrm{g}\left(\mathrm{s}^{*}\right)$ distribution provide insight into the nature of the polydispersity of the solutes. Absorbance peaks that are red shifted indicate the presence of longer and wider rods, while conversely blue shifted peaks are indicative of shorter and thinner rods. The ratio of LSPR to TSPR peaks lower than that of the primary solute suggests the presence of a mixture of rods and spheres. A quantitative analysis of the extracted spectra would require fitting to a simulated spectral model, and is beyond the scope of this manuscript, but is being explored in ongoing research.
The AuNS - AuNR mixture is an artificial representation of what may result from a very poor synthesis execution. Ordinarily a researcher might have only an absorbance spectrum that is distorted from their expectations, but no other means to probe what synthesis byproduct might have been the cause, without laborious TEM imaging that destroys the sample [6]. In one short experiment, the nature of the various solute populations is resolved, and the necessary parameters that one might use for a preparatory centrifuge purification routine are provided. A rigorous evaluation of the hydrodynamic properties of these types of samples requires extensive discussion and is beyond the scope of this study but is the subject of a forthcoming manuscript and will include interpretation of the sedimentation coefficients. It is important to note that, as observed in the experimental data, an AuNR and AuNS may have very similar or even identical sedimentation coefficients, and therefore purification by centrifugation may have limited utility. Furthermore, very large particles will sediment too quickly to collect data in AUC experiments and therefore cannot be resolved. The upper limit of sedimentation coefficients possible with AUC experimentation has not yet been determined.

The NIR-MWL-AUC detector described here will serve equally well for other plasmonic particles with absorbance spectra extending into the NIR. Semiconductors with tunable bandgaps, such as lead-sulfide quantum dots are an example of another class of particles amenable to this technology [33]. Numerous
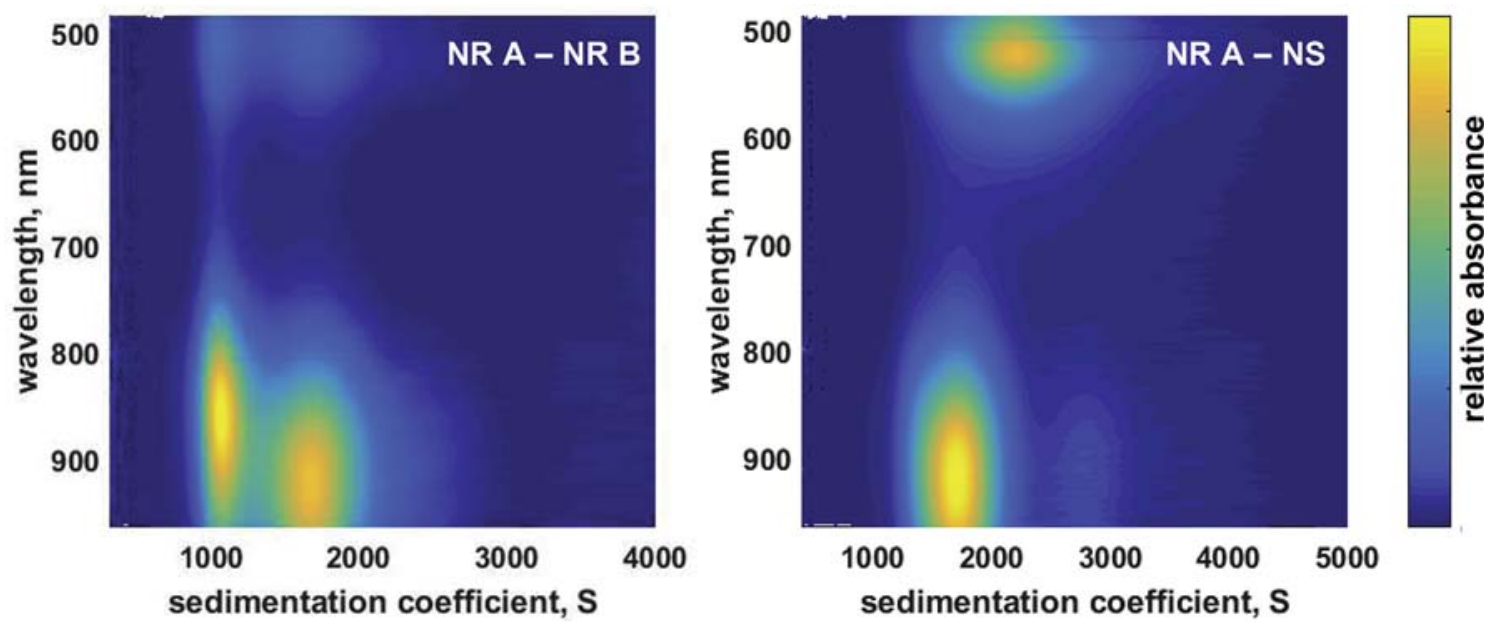

Fig. 8. Results of an AuNR mixture and AuNR-AuNS mixture measured by NIR-MWL-AUC; showing the pseudo 3-dimensional S-spectra from analysis with the WDA method in SedAnal, plotted using Matlab. (Left) Shows the $g\left(s^{*}\right)$ versus wavelength for the mixture of AuNR samples $\mathrm{S}_{\mathrm{A}}$ and $\mathrm{S}_{\mathrm{B}}$, and (right) for the mixture of AuNRs $\mathrm{S}_{\mathrm{A}}$ and AuNSs, $\mathrm{S}_{\mathrm{C}}$. 
investigations have covered a wide array of nanoparticle compositions absorbing in the NIR such as copper and zinc-sulfides, leadselenide, cadmium-telluride, Indium-arsenide, copper-indiumselenide and more complicated multinary compounds [34-40]. NIR absorbing materials are of interest for a host of applications, including sensors, photovoltaic cells, and medical imaging. It is important to note that absorbance in this context refers to extinction arising both from absorbance and scattering events. Conversion from the absorbance signal of a $\mathrm{g}\left(\mathrm{s}^{*}\right)$ to concentration requires knowledge of the extinction spectra of the underlying particle distribution [23]. Because shape information can be directly inferred by sedimentation coefficient and the absorbance spectra maxima, a pathway to this conversion is implied [22].

The NIR spectral signals generated by the system described here are a consequence of the LED array selection, optical coupling and spectrometer configuration. Spectra extending further into the UV or NIR are in principle possible and could be accomplished by the addition of more LED colors, and an appropriately configured spectrometer with NIR optimized gratings and filters. It is interesting to point out that spectrometer gratings with a larger bandwidth tend to, somewhat counter intuitively, have better sensitivity. This is due to the fact that as spectrometer bandwidth is increased, spectral resolution is decreased and a single pixel of the CCD chip receives a wider band-pass. The fiber bundle assembly also has a large influence on the intensity throughput from the LEDs. Some of the LEDs have much higher output, and therefore an equivalent signal can be attained with a smaller fiber core diameter. Future designs should consider a custom fiber bundle, where fiber core diameters are selected to match the relative intensity of the respective LEDs. Custom fiber bundle assemblies also have the possibility to arrange the cores in a linear configuration at the common exit connector. A linear arrangement could replace the slit adapter presently used and would allow for an illumination beam profile matching the slit dimensions of the spectrometer entrance.

The full spectral analysis provided by the WDA method in SedAnal is demonstrated as a convenient method to resolve $\mathrm{g}\left(\mathrm{s}^{*}\right)$ distributions from MWL datasets. For the analysis of MWL broad spectral bandwidth samples involving fitting for sedimentation and diffusion coefficient distributions, and the application of computationally intensive operations such as Monte-Carlo algorithms, the availability of supercomputer methods in programs such as UltraScan3 are very powerful. The two-dimensional spectral analysis (2DSA) and parametrically constrained spectral analysis (PCSA) employed in UltraScan3 have proven especially useful $[15,41,42]$.

\section{Conclusion}

The results presented above demonstrate the basic effectiveness and usefulness of this hardware for NIR-MWL-AUC detection. With proper acquisition settings, sedimentation velocity data can be collected for two cells in an experiment at $3000 \mathrm{rpm}$. Experiments with higher rotor speeds could acquire more signal accumulations per acquisition without increasing scan times. However, this is offset by the narrowing of the open window time available for pulse width modulation. Therefore, limited improvement is expected for higher speeds. More than two cells would result in prohibitively long scan times. To overcome this limitation, a speed ramp acquisition mode is being explored by integration of a National Instruments 'real-time controller'. A speed ramp acquisition mode, while complicated to implement, would allow for data collection of up to 8 cells ( 16 samples) in a single run, and allows analysis of a bigger size range of solutes [31,43]. A simple design modification may be to operate with a speed step profile, with pulse width and integration time adjusted to match the speed steps protocol. Sensible speed steps should be selected to allow for increasing flash accumulations without resulting in prohibitively long scan times.

\section{Acknowledgements}

Funding provided by the Center for Applied Photonics (CAP) at the University of Konstanz and by SFB 1214 (DFG) Project B6.

\section{References}

[1] J. Cao, T. Sun, K.T. Grattan, Gold nanorod-based localized surface plasmon resonance biosensors: a review, Sensor. Actuator. B Chem. 195 (2014) $332-351$.

[2] M.-C. Daniel, D. Astruc, Gold nanoparticles: assembly, supramolecular chemistry, quantum-size-related properties, and applications toward biology, catalysis, and nanotechnology, Chem. Rev. 104 (2004) 293-346.

[3] S. Eustis, M.A. El-Sayed, Why gold nanoparticles are more precious than pretty gold: noble metal surface plasmon resonance and its enhancement of the radiative and nonradiative properties of nanocrystals of different shapes, Chem. Soc. Rev. 35 (2006) 209-217.

[4] J. Pérez-Juste, I. Pastoriza-Santos, L.M. Liz-Marzán, P. Mulvaney, Gold nanorods: synthesis, characterization and applications, Coord. Chem. Rev. 249 (2005) 1870-1901.

[5] N. Li, P. Zhao, D. Astruc, Anisotropic gold nanoparticles: synthesis, properties, applications, and toxicity, Angew. Chem. Int. Ed. 53 (2014) 1756-1789.

[6] V. Sharma, K. Park, M. Srinivasarao, Colloidal dispersion of gold nanorods: historical background, optical properties, seed-mediated synthesis, shape separation and self-assembly, Mater. Sci. Eng. R Rep. 65 (2009) 1-38.

[7] R.P. Carney, J.Y. Kim, H. Qian, R. Jin, H. Mehenni, F. Stellacci, O.M. Bakr, Determination of nanoparticle size distribution together with density or molecular weight by 2D analytical ultracentrifugation, Nat. Commun. 2 (2011) 335.

[8] B. Demeler, T.-L. Nguyen, G.E. Gorbet, V. Schirf, E.H. Brookes, P. Mulvaney, A.a.O. El-Ballouli, J. Pan, O.M. Bakr, A.K. Demeler, Characterization of size, anisotropy, and density heterogeneity of nanoparticles by sedimentation velocity, Anal. Chem. 86 (2014) 7688-7695.

[9] D. Mehn, I.M. Rio-Echevarria, D. Gilliland, M. Kaiser, K. Vilsmeier, P. Schuck, W. Wohlleben, Identification of nanomaterials: a validation report of two laboratories using analytical ultracentrifugation with fixed and ramped speed options, NanoImpact 10 (2018) 87-96.

[10] K. Nontapot, V. Rastogi, J.A. Fagan, V. Reipa, Size and density measurement of core-shell Si nanoparticles by analytical ultracentrifugation, Nanotechnology 24 (2013), 155701.

[11] J. Walter, K. Lohr, E. Karabudak, W. Reis, J. Mikhael, W. Peukert, W. Wohlleben, $H$. Cölfen, Multidimensional analysis of nanoparticles with highly disperse properties using multiwavelength analytical ultracentrifugation, ACS Nano 8 (2014) 8871-8886.

[12] J. Walter, P. Sherwood, W. Lin, D. Segets, W. Stafford, W. Peukert, Simultaneous analysis of hydrodynamic and optical properties using analytical ultracentrifugation equipped with multiwavelength detection, Anal. Chem. 87 (2015) 3396-3403.

[13] S.K. Bhattacharyya, P. Maciejewska, L. Börger, M. Stadler, A.M. Gülsün, H.B. Cicek, H. Cölfen, Development of a Fast Fiber Based UV-vis Multiwavelength Detector for an Ultracentrifuge, 2006, pp. 9-22. Analytical Ultracentrifugation Springer, Berlin Heidelberg.

[14] H. Cölfen, T.M. Laue, W. Wohlleben, K. Schilling, E. Karabudak, B.W. Langhorst, E. Brookes, B. Dubbs, D. Zollars, M. Rocco, B. Demeler, The open AUC project, Eur. Biophys. J. Biophy. 39 (2010) 347-359.

[15] J. Pearson, J. Walter, W. Peukert, H. Cölfen, Advanced multiwavelength detection in analytical ultracentrifugation, Anal. Chem. 90 (2017) 1280-1291.

[16] H. Cölfen, T. Pauck, Determination of particle size distributions with angström resolution, Colloid Polym. Sci. 275 (1997) 175-180.

[17] E. Karabudak, E. Brookes, V. Lesnyak, N. Gaponik, A. Eychmüller, J. Walter, D. Segets, W. Peukert, W. Wohlleben, B. Demeler, H. Cölfen, Simultaneous identification of spectral properties and sizes of multiple particles in solution with subnanometer resolution, Angew. Chem. Int. Ed. 55 (2016) $11770-11774$.

[18] P.K. Jain, K.S. Lee, I.H. El-Sayed, M.A. El-Sayed, Calculated absorption and scattering properties of gold nanoparticles of different size, shape, and composition: applications in biological imaging and biomedicine, J. Phys. Chem. B 110 (2006) 7238-7248.

[19] K.L. Kelly, A.A. Lazarides, G.C. Schatz, Computational electromagnetics of metal nanoparticles and their aggregates, Comput. Sci. Eng. 3 (2001) 67-73.

[20] K.-S. Lee, M.A. El-Sayed, Dependence of the enhanced optical scattering efficiency relative to that of absorption for gold metal nanorods on aspect ratio, size, end-cap shape, and medium refractive index, J. Phys. Chem. B 109 (2005) 
20331-20338.

[21] M.I. Mishchenko, L.D. Travis, A.A. Lacis, Scattering, Absorption, and Emission of Light by Small Particles, Cambridge University Press, 2002.

[22] R.D. Near, S.C. Hayden, M.A. El-Sayed, Thin to thick, short to long: spectra properties of gold nanorods by theoretical modeling, J. Phys. Chem. C 117 (2013) 18653-18656.

[23] S.W. Prescott, P. Mulvaney, Gold nanorod extinction spectra, J. Appl. Phys. 99 (2006), 123504

[24] G. González-Rubio, P. Díaz-Núñez, A. Rivera, A. Prada, G. Tardajos, J. GonzálezIzquierdo, L. Bañares, P. Llombart, L.G. Macdowell, M.A. Palafox, Femtosecond laser reshaping yields gold nanorods with ultranarrow surface plasmon resonances, Science 358 (2017) 640-644.

[25] Y. Sun, Y. Xia, Shape-controlled synthesis of gold and silver nanoparticles, Science 298 (2002) 2176-2179.

[26] J. Walter, K. Löhr, E. Karabudak, W. Reis, J. Mikhael, W. Peukert, W. Wohlleben, H. Cölfen, Multidimensional analysis of nanoparticles with highly disperse properties using multiwavelength analytical ultracentrifugation, ACS Nano 8 (2014) 8871-8886.

[27] C.A. Palmer, E.G. Loewen, Diffraction Grating Handbook, Newport Corporation, New York, 2005.

[28] H. Cölfen, P. Husbands, S. Harding, Alternative Light Sources for the Schlieren Optical System of Analytical Ultracentrifuges, Springer, 1995, pp. 193-198. Analytical Ultracentrifugation.

[29] W.F. Stafford, E.H. Braswell, Sedimentation velocity, multi-speed method for analyzing polydisperse solutions, Biophys. Chem. 108 (2004) 273-279.

[30] H. Fujita, Foundations of Ultracentrifugal Analysis, Wiley, 1975.

[31] W. Mächtle, High-resolution, submicron particle size distribution analysis using gravitational-sweep sedimentation, Biophys. J. 76 (1999) 1080-1091.

[32] J. Pearson, F. Krause, D. Haffke, D. Demeler, K. Schilling, H. Cölfen, Next generation AUC adds a spectral dimension: development of multiwavelength detectors for the analytical ultracentrifuge, Meth. Enzymol. 562 (2015) 1-26.

[33] J. Zhang, J. Gao, E.M. Miller, J.M. Luther, M.C. Beard, Diffusion-controlled synthesis of $\mathrm{PbS}$ and $\mathrm{PbSe}$ quantum dots with in situ halide passivation for quantum dot solar cells, ACS Nano 8 (2013) 614-622.
[34] S. Kim, A.R. Marshall, D.M. Kroupa, E.M. Miller, J.M. Luther, S. Jeong, M.C. Beard, Air-stable and efficient PbSe quantum-dot solar cells based upon ZnSe to PbSe cation-exchanged quantum dots, ACS Nano 9 (2015) 8157-8164.

[35] J.-Y. Kim, J. Yang, J.H. Yu, W. Baek, C.-H. Lee, H.J. Son, T. Hyeon, M.J. Ko, Highly efficient copper-indium-selenide quantum dot solar cells: suppression of Carrier recombination by controlled ZnS overlayers, ACS Nano 9 (2015) $11286-11295$.

[36] H. Nishi, K. Asami, T. Tatsuma, CuS nanoplates for LSPR sensing in the second biological optical window, Opt. Mater. Express 6 (2016) 1043-1048.

[37] M.G. Panthani, V. Akhavan, B. Goodfellow, J.P. Schmidtke, L. Dunn, A. Dodabalapur, P.F. Barbara, B.A. Korgel, Synthesis of CuInS2, CuInSe2, and $\mathrm{Cu}$ (In x Ga1-x) Se2 (CIGS) nanocrystal "inks" for printable photovoltaics, J. Am. Chem. Soc. 130 (2008) 16770-16777.

[38] M.D. Regulacio, M.-Y. Han, Multinary I-III-VI2 and I2-II-IV-VI4 semiconductor nanostructures for photocatalytic applications, Accounts Chem. Res. 49 (2016) 511-519.

[39] H. Shen, H. Wang, X. Chen, J.Z. Niu, W. Xu, X.M. Li, X.-D. Jiang, Z. Du, L.S. Li, Size-and shape-controlled synthesis of CdTe and PbTe nanocrystals using tellurium dioxide as the tellurium precursor, Chem. Mater. 22 (2010) 4756-4761.

[40] J.P. Zimmer, S.-W. Kim, S. Ohnishi, E. Tanaka, J.V. Frangioni, M.G. Bawendi, Size series of small indium arsenide- zinc selenide core- shell nanocrystals and their application to in vivo imaging, J. Am. Chem. Soc. 128 (2006) 2526-2527.

[41] G. Gorbet, J. Pearson, A. Demeler, H. Cölfen, B. Demeler, Next generation AUC: analysis of multi-wavelength analytical ultracentrifugation data, Meth. Enzymol. 562 (2015) 27-47.

[42] J. Zhang, J.Z. Pearson, G.E. Gorbet, H. Cölfen, M.W. Germann, M.A. Brinton, B. Demeler, Spectral and hydrodynamic analysis of west nile virus RNAprotein interactions by multiwavelength sedimentation velocity in the analytical ultracentrifuge, Anal. Chem. 89 (2016) 862-870.

[43] J. Walter, D. Segets, W. Peukert, Extension of the deep UV-capabilities in multiwavelength spectrometry in analytical ultracentrifugation: the role of oil deposits, Part. Part. Syst. Char. 33 (2016) 184-189. 